\title{
Review Article \\ The Role of the Mitochondrial Genome in Ageing and Carcinogenesis
}

\author{
Anna M. Czarnecka ${ }^{1}$ and Ewa Bartnik ${ }^{2,3}$ \\ ${ }^{1}$ Laboratory of Molecular Oncology, Department of Oncology, Military Institute of Medicine, ul. Szaserów 128, 01-141 Warsaw, Poland \\ ${ }^{2}$ Institute of Genetics and Biotechnology, Faculty of Biology, University of Warsaw, Pawinskiego 5a, 02-106, Warsaw, Poland \\ ${ }^{3}$ Institute of Biochemistry and Biophysics, Polish Academy of Sciences, Pawinskiego 5a, 02-106 Warsaw, Poland
}

Correspondence should be addressed to Ewa Bartnik, ebartnik@igib.uw.edu.pl

Received 29 October 2010; Accepted 3 January 2011

Academic Editor: Alberto Sanz

Copyright (C) 2011 A. M. Czarnecka and E. Bartnik. This is an open access article distributed under the Creative Commons Attribution License, which permits unrestricted use, distribution, and reproduction in any medium, provided the original work is properly cited.

Mitochondrial DNA mutations and polymorphisms have been the focus of intensive investigations for well over a decade in an attempt to understand how they affect fundamental processes such as cancer and aging. Initial interest in mutations occurring in mitochondrial DNA of cancer cells diminished when most were found to be the same mutations which occurred during the evolution of human mitochondrial haplogroups. However, increasingly correlations are being found between various mitochondrial haplogroups and susceptibility to cancer or diseases in some cases and successful aging in others.

\section{Introduction}

Mitochondria play essential and diverse roles in the physiology of eukaryotic cells. These structures are not only indispensable for ATP production and participate in numerous intermediate metabolic reactions but also play a central role in calcium homeostasis, apoptosis, cell signaling, and differentiation. Impairment of mitochondrial functions has been implicated in a wide variety of human pathologies including cancer and age-related diseases such as type II diabetes mellitus (DM II), Alzheimer's disease, degenerative arthritis, ischemic heart disease, and age-related macular degeneration [1-4].

Until recently aerobic glycolysis of cancer cells received little attention in oncology despite being one of the first observations made in the field [5]. Recent progress in the understanding of cancer molecular genetics, biochemistry, and proteomics has renewed interest in "mitochondrial oncology" $[2,4]$. It is now widely understood that, unlike most normal cells, cancer cells are dependent on glycolysis for energy production and that an energetic switch is an indispensible step in malignant transformation [6], since aerobic glycolysis confers a growth advantage to tumor cells [7] and disrupted OXPHOS provides a proliferative advantage [8]. New attempts to analyze the significance of mitochondria in oncology encompass the increasingly important debate whether mtDNA mutations or polymorphisms are actually the cause or an effect of tumor progression. A growing number of reports support the hypothesis that mitochondrial functions are profoundly altered in transformed cells and that mtDNA polymorphisms are important for the processes of cell transformation $[3,4$, $9,10]$.

Somatic mtDNA mutations are a general phenomenon occurring during cell transformation and ageing. In the last ten years mtDNA mutations have been found in solid tumors including carcinomas and sarcomas, and also leukemias and lymphomas $[1,3,11-13]$. At the same time reports of premature aging in mutant mice with greatly increased rates of mitochondrial DNA mutagenesis-so-called "mitochondrial mutator mice"-confirmed that accumulation of mtDNA mutations is a key mechanism of aging [14]. Comprehensive scanning of mtDNA in cancer cells detected functionally relevant point mutations [15-17], but also specific mtDNA polymorphisms in polypeptide-encoding genes and the Dloop were found in as many as $50 \%$ of cancer patients 
[18-20]. MtDNA mutations and polymorphisms can cause alterations in the encoded proteins $[21,22]$ and in consequence may compromise the respiratory chain function and stimulate abundant ROS production [15, 23]. Proteins with altered sequences as the result of somatic mtDNA mutations have been shown to have disturbed assembly and stability and therefore defective structure of respiratory chain complexes leading to OXPHOS deficiency [21, 22, 24, 25]. Such mutations could also affect cells during aging.

An analogous correlation has been proposed for cells with specific rare mtDNA polymorphisms [26-30]. Thus, the different mtDNA sequences reported in a variety of human cancer cells are thought to contribute to oxidative respiratory malfunction. In particular a few reports confirm that mtDNA genome sequence heterogeneity is the cause of subsequent mitochondrial function alterations including OXPHOS deficiency, ROS overproduction, and finally upregulation of signaling pathways associated with cell maturation, proliferation, and cell death $[12,25,31,32]$. The codependence of cancer growth rate, metastatic potential and mtDNA mutations has recently been established in a mouse model [15]. Moreover, the apparent conundrum of whether mtDNA mutations found in tumors are a cause or a consequence of the carcinogenic process has recently also been partially solved after the demonstration that pathogenic mtDNA mutations lead to excessive reactive oxygen species signaling [33, 34], diminish cellular apoptotic potential [35], initiate mitochondria-nucleus signaling that promotes a cellular invasive phenotype [36-38], regulate epigenetic modifications in the nucleus [10], and affect nucleotide pools and chromosomal stability [39]. Although numerous mtDNA polymorphisms and mutations have been identified in various tumors, the pathogenic implications (cell growth or transformation promotion? metastatic potential promotion?) of many of these mtDNA variants remain unclear and need critical evaluation in order to avoid pitfalls, misinterpretations, and erroneous conclusions [40, 41].

Comprehensive analysis by Bandelt et al. [41-43] shows that oncology, forensic science, population genetics, and clinical literature on mtDNA is often compromised by unsatisfactory results in many studies, including flawed sequence data, artificial recombinant sequences or interpretative shortcomings [43-45]. In particular phantom mutations and phantom mutation hotspots lead to misidentification of pathologic somatic mutations and to misinterpretations in all kinds of aspects of clinical mtDNA studies [46, 47]. Many listed mutations when reanalyzed were found to actually be polymorphisms, including polymorphisms known to define major haplogroups. It should be stressed that those artifacts have been inadvertently regarded as novel and pathogenic. Bandelt et al. proposed a paradigm of evaluation of sequence quality and detection of potential problems when inferring the pathogenic status of an mtDNA mutation to avoid false positive results [48]. Some authors have also called for stringent quality control of mtDNA data by haplogroupdirected database comparisons $[47,49]$. Therefore, in this paper we mainly focus on mtDNA polymorphisms and mitochondrial haplogroups and their role in cancer and aging.

\section{Mitochondrial DNA Polymorphisms in Cancer}

The use of mitochondrial DNA polymorphisms as biomarkers is rapidly expanding in metabolic disease diagnostics, the analysis of human migration patterns, and human identification in forensic sciences [2, 11, 12]. Although somatic mtDNA mutations have been reported in many types of cancer, very few reports have documented the prevalence of inherited mitochondrial DNA polymorphisms in cancer patients and its comparison to healthy control populations.

The first interesting and widely investigated mtDNA polymorphism in the cancer field was A10398G, first described as causative factor in breast cancer development [50-52]. In particular we have shown the abundance of the $10398 \mathrm{G}$ polymorphism in a Polish breast cancer cohort. $23 \%$ of patients with biopsy-proven breast cancer carried the $10398 \mathrm{G}$ variant, while in the healthy group only $3 \%$ $(P=.0008)$. This polymorphism is of great interest as 10398 locus in mtDNA is highly polymorphic. While the revised Cambridge reference sequence [53] lists the wild type base as A, the alternative base $(\mathrm{G})$ is also prevalent in many populations [54]. Amongst 2704 complete coding region sequences reported in the $\mathrm{mtDB}$ database, the base is $\mathrm{A}$ in $54 \%$ (1461), $\mathrm{G}$ in $46 \%$ (1242), and $T$ in 1 case $[54,55]$. Moreover, analyses of this polymorphism in the clinical context yielded contradictory findings. The 10398A appears to increase the risk of Parkinson's disease [56], Alzheimer's disease [57], and amyotrophic lateral sclerosis [58], but in contrast it protects against cardiomyopathy in patients with Friedreich's ataxia [59]. At the same time Canter et al. found an increased frequency of the 10398A in African-American women with invasive breast cancer [60]. In contrast, in another study, the 10398A frequency was not increased in an African American breast cancer cohort when compared with controls [50]. The significant difference reported by us between $10398 \mathrm{G}$ distribution in breast cancer patients and breast cancer-free women with negative family history seems to prove the unique role of the A10398G polymorphism in breast cancer predisposition. We believe that A10398G might be considered as a contributing factor in carcinogenesis and as variant modifying the risk of developing breast cancer $[19,61]$. In contrast, inheritance of 10398A has been postulated to increase incidence and severity of prostate cancer in African-American men as compared with other ethnic groups [52]. Concerning the pathophysiological role of A10398G it must be underlined that the amino acid with $10398 \mathrm{~A}$ is threonine, and with 10398G alanine. Amongst 61 analyzed species (including humans), the amino acid at position 114 is threonine in $44 \%$, and alanine in 30\% and a different amino acid in 26\% [62]. The substitution of threonine-a polar, neutral amino acid with hydropathy index -0.7 for alanine-a nonpolar, neutral amino acid with hydropathy index of 1.8 [63] —is significant and relatively nonconservative as reflected by the Grantham value of 58 [64]. The methyl group of alanine is nonreactive and is thus almost never directly involved in protein function and bonding $[65,66]$. 
Concerning other mtDNA loci, multiple interesting mtDNA polymorphisms have been reported in prostate [67], thyroid [68], and oral cancer cases [69] with breast cancer as the most widely explored research topic $[20,27,70-$ 74]. It was shown that breast cancer patients with multiple $m t D N A$ D-loop (CA)(n) polymorphisms have significantly poorer disease-free survival than those with one copy of the mtDNA D-loop (CA)(n) polymorphism [70]. mtDNA variation, particularly in the D310 segment, was indicated to be a causative factor of breast cancer development. In particular the C16189T polymorphism seems to be of potential pathophysiological impact as it has been associated with susceptibility to dilated cardiomyopathy (DCM) [75], insulin resistance, and development of DM II in adult life and vascular pathologies including lacunar cerebral infarction and cardiovascular diseases [76]. In a breast cancer cohort study we detected a significantly greater incidence of mtDNA polymorphisms T239C, A263G, and C16207T and a significant lower incidence of A73G, C150T, T16183C, T16189C, C16223T, and T16362C compared to controls [27]. In breast cancer cohort NADH-dehydrogenase mtDNA encoded genes were also rich in polymorphisms, in particular very rare polymorphisms such as A4727G, G9947A, A10044G, A10283G, T11233C, and C11503T [19].

At the same time we have also reported multiple mtDNA polymorphisms in vulvar cancer cases, in particular 19 specific uncommon polymorphisms and one polymorphism not reported previously (C498delC). In vulvar cancer cases overrepresented polymorphisms included C16192T, C16256T and C16270T, what is interesting is that, all are specific for haplogroup $\mathrm{U}$, whereas the $16223 \mathrm{~T}$ polymorphism characteristic for haplogroups I, W, and X was underrepresented $[28,54]$. In all the studies, including breast cancer, endometrial adenocarcinoma, and vulvar carcinoma, polymorphisms were predominantly found in mtDNA hypervariable regions HV1 (16024-16383) and HV2 (57-333) [26-28].

In the case of hepatocellular carcinoma (HCC), polymorphisms including 16266T, 16293G, 16299G, 16303A, 242T, $368 \mathrm{G}$, and $462 \mathrm{~T}$ were associated with increased risk for alcohol-dependent HCC. At the same time the 523A/del was associated with increased risks of both alcohol-dependent, and independent HCC. Moreover, 489T/152T, 489T/523A, and $489 \mathrm{~T} / 525 \mathrm{C}$ haplotypes were significantly reduced in HBV-positive HCC patients in comparison to HBV-negative patients. Haplotypes 489C and 152T, 249A, 309C, 523Del, or 525Del were associated significantly with an increase of alcohol-HCC risk [77].

6221C and 7389C polymorphisms were significantly associated with prostate cancer $(P<.05)$ and in strong linkage disequilibrium with each other $(r(2)>0.6)$ in African-American men [78], while no specific mtDNA polymorphisms appeared to have a significant effect on a predisposition to prostate cancer in the Korean population [79].

In summary we and others have hypothesized that patients bearing some of the common mitochondrial polymorphisms are at higher risk of cancer development, with a particularly interesting role of haplogroup-specific
mtDNA polymorphisms. Moreover, it might be tempting to suggest that in cancer patients a similar situation is found as that reported by Lehtonen et al. [80] for patients with sensorineural hearing impairment-that is, in both cases, affected persons have more rare substitutions in their mitochondrial DNA than the healthy control population. Similar results have been obtained for other diseases related to mitochondria [81]. It is difficult to explain this at present, but possibly these rare mutations have an effect on mitochondrial function making persons in whom they are present more susceptible to certain diseases.

\section{Mitochondrial Mutations and Polymorphisms in Aging}

Numerous papers have analyzed the increased occurrence of mitochondrial mutations and deletions in aging cells in various organisms [82-84]. The fact that older cells in various tissues, especially in postmitotic ones, accumulate both point mutations and deletions in mitochondrial DNA is undisputed, but there have been a number of problems interpreting these results. First, relatively high percentages of mutations are known to be required to affect the respiratory chain, on the basis of data from patients with mitochondrial diseases. Second, the correlation of point mutations and deletions with aging is not proof of causality—only of coexistence. Another problem which has been controversial is what the effects of the mutations are-do they cause a vicious cycle of ROS release, as was postulated by Harman [85], with more and more new mutations leading inevitably to cell death?

In the last years two models have resolved some, but not all of the problems which have been raised in studies of aging and mitochondria. The first was mentioned abovethe mutator mouse, obtained independently by two groups $[86,87]$ with a mutated mitochondrial DNA polymerase which led to accumulation of large numbers of mutations in the mitochondrial DNA. The mice showed symptoms of premature aging - and, as the mutations came before the aging this was proof of causality. There are still, however, some problems with the interpretation of the results obtained with these models. Why are heterozygous mice which accumulate a high number of mutations healthy? Are deleted mtDNA molecules the cause of aging? Do they occur? The different techniques used and different results obtained by the two groups make it difficult to analyze what exactly is happening during the aging process, but some things do appear to be clear-there are numerous point mutations, they lead to cell death and eventually aging, and all this takes place without any excessive production of ROS [88-90].

The second result was perhaps less spectacular than those obtained with the mutator mouse but is very important for solving the problem of whether and how low levels of mitochondrial DNA mutations can affect the functioning of cells. Dufour et al. [91] found that a mitochondrial respiratory chain deficiency in neurons which was caused by a nuclear mutations when present in only $20 \%$ of 
the mitochondria caused degeneration of adjacent neurons. This points to a solution of the problem that the levels of mutations found in aging tissues are too low.

Thus, mitochondrial mutations are now generally believed to be involved in the aging process. The involvement of ROS will be discussed in a separate section as will the effects of mitochondrial polymorphisms - through their grouping in haplogroups.

\section{Mitochondrial Haplogroups in Cancer and Aging}

Human mtDNA is highly polymorphic. On the basis of certain SNPs present in mtDNA, the human population can be divided into haplogroups. Analysis of their distribution among the population enables reconstruction of ancient human migrations. Mutations creating certain haplogroups (that became polymorphisms over time) were suggested to affect coupling efficiency of the electron transport chain, enabling adaptation to life in different climatic conditions [2]. It is estimated that around $25 \%$ of mtDNA polypeptide sequence variation, $10-20 \%$ of tRNA variation, and at least some of rRNA variation contributes to this effect.

MtDNA haplogroup variation seems to confer genetic susceptibility for several conditions, as indicated by numerous studies. Mitochondrial genetic background was shown to affect the risk of visual failure caused by Leber's Hereditary Optic Neuropathy (LHON), as mutation occurrence in patients with haplogroup J or K correlated with an elevated risk of developing the condition, whereas haplogroup $\mathrm{H}$ had a protective effect $[2,11,92,93]$. A similar effect was reported for haplogroups $\mathrm{M} 8 \mathrm{a}$ and $\mathrm{M} 7 \mathrm{~b}$ that diminished and enhanced, respectively, clinical manifestation of LHON symptoms in a Chinese population [94]. Haplogroup H1 was shown to have protective effects on ischemic stroke risk among Portuguese patients [95]. Parkinson's disease (PD) was less frequent among $\mathrm{K}$ haplogroup individuals in the Italian population [96], whereas a study on Polish $\mathrm{PD}$ patients reported a protective effect of haplogroup $\mathrm{J}$ in males [97]. JTWIX supercluster background was associated with a higher number of nonsynonymous mutations in complex I genes and an increased risk of PD. At the same time haplogroups $U$ and $\mathrm{K}$ seem to neutralize the harmful effect of the APOE4 variant in Alzheimer's disease [98] and be connected with psychiatric disorders [99]. Moreover, polymorphisms characteristic for haplogroup $\mathrm{J}$ and $\mathrm{M}$ were shown to be associated with longevity in European and Japanese populations, respectively [100]. On the other hand, haplogroup J was reported to confer susceptibility to type II diabetes [101].

In the cancer field, significant increased risk for breast cancer development was reported for haplogroup K [102]. Our analysis revealed that mitochondrial haplogroup distribution in patients with breast cancer greatly differs from cancer-free controls and the general Polish population. Haplogroup I was overrepresented in individuals with cancer (14\% versus $3 \% ; P=.017$ ) in comparison to the general Polish population, and if the breast cancer cohort was compared to cancer-free centenarians, haplogroup $\mathrm{H}$ was underrepresented (38\% versus $58 \%$; $P=.019$ ) [27]. It was shown that haplogroup $M$ individuals have an increased risk of breast cancer compared with haplogroup $\mathrm{N}$ in China [103]. In a specific Chinese population, patients with mtDNA haplogroup $M$ exhibited an increased risk of breast cancer development $(P=.040)$ and that this haplogrouprisk effect was even more pronounced in a subhaplogroup of M, subhaplogroup D5 $(P=.030)$ [104].

Concerning other types of cancer not as much research was done as in the case of breast cancer; nevertheless, it was proven that haplogroup D4a is associated with an increased risk of thyroid cancer development $(P=.028)$ [104]. The inheritance of mitochondrial haplogroup $U$ was shown to be associated with an approximately 2 -fold increased risk of prostate cancer and 2.5-fold increased risk of renal cancer in white North American individuals and it was indicated that individuals with this mitochondrial haplotype are in a high-risk group. Because mitochondrial haplogroup $U$ is found in $9.35 \%$ of the white United States population, more than 20 million individuals were shown to be in a high risk group [105]. On the other hand, in a Middle European population mitochondrial haplogroup frequencies did not differ significantly between patients with prostate cancer and control population, implying no impact of inherited mitochondrial DNA variation on predisposition to PC [106]. Esophageal cancer (EC) predisposition was investigated in Taihang Mountain and Chaoshan areas in China. In this study D5 haplogroup was associated with EC at the general population level in the Taihang Mountain area and in women below 60 years of age in the Chaoshan area. It was also shown that D4a and D5 haplogroups might be risk markers of EC development, in particular D4a and D5 for the Taihang Mountain area and D and D5 for the Chaoshan area [107].

Mitochondrial haplogroups were also in the focus of gynecological oncology. In vulvar cancer patients, we again found significant differences with underrepresentation of haplogroup $\mathrm{H}$ in the cancer cohort. Moreover, in the comparison with the general Polish population a trend towards haplogroup $\mathrm{U}$ and $\mathrm{K}$ overrepresentation was also noticed (44 versus 19\%, $P=.009$ ) [28]. The underrepresentation of haplogroup $\mathrm{H}(P=.023)$ in vulvar cancer patients was particularly interesting. Haplogroup $\mathrm{H}$ is marked by the T7028C polymorphism. Therefore, if haplogroup $\mathrm{H}$ is underrepresented, 7028T polymorphism is overrepresented. For vulvar cancer the C7028T RFLP test has Odds Ratio (OR) and Diagnostic Odds Ratio = 3.11 and indicates Relative Risk $(\mathrm{RR})=2.43$ in comparison to the control (cancer-free centenarians) [28]. A similar trend was reported for endometrial adenocarcinoma cases. Analysis revealed that the endometrial carcinoma population haplogroup distribution differs from the Polish population with haplogroup $\mathrm{H}$ being strongly underrepresented $(P=$ $.003)$. In this group patients with endometrial adenocarcinoma had the T7028C $12 \%$ of the time while the general Polish population carried the T7028C polymorphism $42 \%$ of the time. As a result one had a 5.53 higher chance to be $\mathrm{T} 7028 \mathrm{C}$ carrier and not develop cancer with specificity of prediction as high as $0.775(0.755-0.815)$ [26]. In both 
vulvar and endometrial cancer cases Diagnostic Odds Ratio of C7028T test was in the range of 3-6, and therefore may provide a strong clue in investigations of etiology [108]. In another study patients with endometrial cancers clustered in haplogroup $\mathrm{D}$ with a significantly higher frequency [109].

In summary we believe that, because the patterns of germline polymorphisms in cancer patients and controls are different, it is possible that the inheritance of specific mitochondrial genotypes predisposes individuals to cancer $[110,111]$. Moreover, the co-occurrence of multiple inherited mtDNA SNPs may influence the disease phenotype as shown in the case of $12308 \mathrm{G}$ and $10398 \mathrm{G}$ in breast cancer [61] or G10680A and T14484C in LHON [112]. One polymorphism may have a modifying role for increasing the penetrance and expressivity of other mtDNA genome variants. In particular, the mitochondrial haplotypes may play a synergistic role in the development of cancer as is the case of vision loss in the families carrying the LHONassociated primary mtDNA mutations. In LHON patients the mitochondrial haplotype has been shown to influence the clinical expression [94].

The relationship between mitochondrial haplogroups and aging in humans is based on analysis of centenarians in various populations and appropriate control groups; in some populations certain haplogroups were found to be overrepresented in relation to controls [113]. A very attractive hypothesis has been proposed by Wallace [2] that successful aging is due to uncoupling of the electron chain. In brief, persons with more uncoupled haplogroups would produce more heat (an adaptation to colder climates), be more calorie restricted, and thus age better, but also be more susceptible to certain mitochondrial diseases. This has not been confirmed directly, as comparison of uncoupling in cell lines with identical genetic backgrounds repopulated with mitochondria from haplogroups $\mathrm{H}$ and $\mathrm{T}$ did not show any differences [114], but there are some results concerning human lifespan under conditions of good and poor supply which indicate that for instance persons with haplogroup $\mathrm{H}$ live longer than persons with haplogroup U under conditions when the food supply is limited [115]. Differences in transcription between haplogroups have also been observed [116], though a direct difference in uncoupling was not analyzed. There are also some data indicating that mice with more uncoupled mitochondria live longer than mice without the uncoupling [117]. However, there is a problem that few of these association studies would fulfill the criteria proposed by Samuels et al. [118] as the analyzed groups were often too small. Thus, the conclusion would be that there are interesting suggestions of correlations of mitochondrial haplogroups and aging, but the evidence is still too weak and may indeed remain that way. On the other hand, there are new papers indicating differences between some haplogroups for example, between haplogroups $\mathrm{H}$ and UK in respect to among others oxygen consumption and protein synthesis [119] and persons with haplogroup $\mathrm{H}$ have been shown to have higher mitochondrial oxidative damage than those with haplogroup $\mathrm{J}$ [120], and it is possible that this problem will be solved in the future.

\section{Reactive Oxygen Species in Carcinogenesis and Ageing}

Multiple papers have indicated that oxidative stress is central in the pathogenesis of a wide variety of diseases including cancer and a special role in mitochondria-related ageing has been attributed to reactive oxygen species (ROS) $[1,2,121]$. The mitochondrial respiratory chain is the major source of ROS, which in excess obviously destroys mtDNA, in turn contributing to the promotion of a vicious cycle of cancer development and possibly aging. The constant generation of ROS within the mitochondria and the increased oxidative stress in cancer cells may cause further damage to the electron transport chain, and amplify respiratory malfunction and cancer dependency on glycolysis. It is ROS production that increases when electron transport is reduced or inhibited, as in the case of low aerobic respiration rate resulting from mtDNA mutations and aberrant OXPHOS protein structure [122]. The main sources of mitochondrial ROS are complex I and complex III since mitochondrial generation of ATP through oxidative phosphorylation involves formation of $\mathrm{O}_{2}^{-}$and subsequently derived ROS such as hydrogen peroxide and $\cdot \mathrm{OH}$. This is the case of complex I flavin active site, complex I and complex III iron-sulfur centers and ubisemiquinone transferring electrons to oxygen and giving rise to superoxide anions. At the same time if the respiratory chain is inhibited downstream of complex III (as in the case of some mtDNA mutations), electrons coming from succinate oxidation also increase superoxide anion generation [123]. Subsequently, oxidative stress develops and ROS scavenging is overloaded [124, 125].

The scenario that emerges from ROS, mtDNA mutations and cancer studies is rather complex [124, 125], since different mtDNA mutations do not result in the same profile in terms of ROS production $[23,40]$. For example, it has been proven that only mtDNA mutations that affect the function of complex I and III, but not COX mutations, are triggers of ROS production [33] and that only specific mutations in complex I genes with subsequent ROS overproduction may promote tumor growth [17] and cancer metastases [15]. In contrast Zielonka and Kalyanaraman [126] recently have proven that Ishikawa et al. [15] failed to show evidence for formation of superoxide and hydrogen peroxide, presumed to be generated from complex I deficiency associated with mtDNA mutations in metastatic cells.

The involvement of ROS and mitochondria in cancer appears to be generally accepted [127]. On the other hand, the role of ROS in aging appears to be less well established, in spite of the very long period in which it has been the fundamental hypothesis for much of aging research. During the last years a number of papers have suggested that aging does not require ROS formation [128] and the free radical theory of aging has been increasingly under attack and appears to be no longer the major theory explaining this phenomenon [129, 130]. However, as is often true for fields as complex as aging, the situation is not simple and discussions as to the role of ROS will continue. This paper deals essentially with mammals; however, recent work on Drosophila melanogaster has shown that mitochondrial 
DNA controls longevity in females regardless of ROS production, and moreover a number of predictions of the mitochondrial free radical theory of aging were not verified [131].

Interpretation of many results is quite complex. For instance, Schriner et al. [132] have shown that overexpression of catalase in mitochondria in mice extends their lifespan. This could be construed as evidence that ROS removal affects longevity. The overexpression of this enzyme not only alleviates aging symptoms in the heart in normal mice [133] but also in the mutator mouse model [134]. Thus, the question is, if the mutator mouse ages without ROS production, what does the catalase do in the heart of these mice? Dubec et al. [135] on the basis of a mouse model with a proofreading-deficient mitochondrial polymerase targeted only to the heart proposed that cell death and cardiomyopathy are due to apoptosis caused by rare mutations in mitochondrial DNA leading to the release of an apoptosis-promoting peptide.

Where does this leave mitochondria? There are still numerous data indicating their involvement in the aging process-from model organisms $[129,136]$ to humans $[1,2]$. Moreover, the exact mechanisms through which mitochondrial function affects aging is still unknown, what does appear to be clear is that neither are ROS required for aging nor does their production necessarily cause it.

\section{Summary and Perspectives}

Precise understanding of the molecular mechanisms whereby specific mtDNA polymorphisms predispose to cancer is currently lacking. Although the mechanisms of generation and functional impact of mtDNA polymorphisms are still not clear, there is a high incidence and broad distribution in human cancers making them a potential marker for cancer detection, but the role of mtDNA polymorphisms in the maintenance of the tumor cell phenotype or in tumorigenesis remains to be elucidated [2, 3, 137, 138]. Although it is generally believed that polymorphisms may have pathogenic potential, it remains to be established whether they contribute to neoplastic transformation by changing cellular energy capacities, increasing mitochondrial oxidative stress, or modulating apoptosis. The significance of each individual polymorphism for mitochondrial function and tumorigenesis is unknown and only functional analysis in relation to cell behavior, proliferation, and apoptosis can determine the significance of these polymorphisms and their association with tumorigenesis. We believe that polymorphisms in mtDNA, both in the D-loop and in the coding region (including haplogroup-specific positions), may cause subtle differences in the encoded protein structure and function $[4,12,139]$. Particular mtDNA genotypes may predispose an individual to an earlier onset of degenerative cellular processes, such as the accumulation of somatic mtDNA variation, decline in OXPHOS capacity or faster cancer progression, as shown in transmitochondrial cybrids [140]. The mtDNA haplotype may also influence its further mutagenesis, as has been shown for haplogroup J that predisposes the mitochondrial genome to mutate at locus 14484, possibly through near-neighbor effects as previously described to occur during mtDNA evolution [141]. All these phenomena may affect both cancer and aging.

\section{Acknowledgment}

This work was supported by Grant N N401 049238 from the Polish Ministry of Health and Higher Education.

\section{References}

[1] D. C. Wallace, "Mitochondrial DNA mutations in disease and aging," Environmental and Molecular Mutagenesis, vol. 51, no. 5, pp. 440-450, 2010.

[2] D. C. Wallace, "A mitochondrial paradigm of metabolic and degenerative diseases, aging, and cancer: a dawn for evolutionary medicine," Annual Review of Genetics, vol. 39, pp. 359-407, 2005.

[3] A. M. Czarnecka and E. Bartnik, "Mitochondrial DNA mutations in tumors," in Cellular Respiration and Carcinogenesis, S. P. Apte and R. Sarangarajan, Eds., pp. 1-12, Humana Press, New York, NY, USA, 2009.

[4] A. M. Czarnecka et al., "Cancer as a "Mitochondriopathy"', Journal of Cancer Molecules, vol. 3, no. 3, pp. 71-79, 2007.

[5] O. Warburg, "On the origin of cancer cells," Science, vol. 123, no. 3191, pp. 309-314, 1956.

[6] E. Gottlieb and I. P. M. Tomlinson, "Mitochondrial tumour suppressors: a genetic and biochemical update," Nature Reviews Cancer, vol. 5, no. 11, pp. 857-866, 2005.

[7] R. J. Gillies and R. A. Gatenby, "Hypoxia and adaptive landscapes in the evolution of carcinogenesis," Cancer and Metastasis Reviews, vol. 26, no. 2, pp. 311-317, 2007.

[8] H. Simonnet, N. Alazard, K. Pfeiffer et al., "Low mitochondrial respiratory chain content correlates with tumor aggressiveness in renal cell carcinoma," Carcinogenesis, vol. 23, no. 5, pp. 759-768, 2002.

[9] M. Kulawiec, K. M. Owens, and K. K. Singh, "Cancer cell mitochondria confer apoptosis resistance and promote metastasis," Cancer Biology and Therapy, vol. 8, no. 14, pp. 69-76, 2009.

[10] D. J. Smiraglia, M. Kulawiec, G. L. Bistulfi, S. G. Gupta, and K. K. Singh, "A novel role for mitochondria in regulating epigenetic modification in the nucleus," Cancer Biology and Therapy, vol. 7, no. 8, pp. 1182-1190, 2008.

[11] M. Brandon, P. Baldi, and D. C. Wallace, "Mitochondrial mutations in cancer," Oncogene, vol. 25, no. 34, pp. 46474662, 2006.

[12] A. M. Czarnecka, P. Golik, and E. Bartnik, "Mitochondrial DNA mutations in human neoplasia," Journal of Applied Genetics, vol. 47, no. 1, pp. 67-78, 2006.

[13] A. M. Czarnecka, W. Kukwa, T. Krawczyk, A. Scinska, A. Kukwa, and F. Cappello, "Mitochondrial DNA mutations in cancer -from bench to bedside," Frontiers in Bioscience, vol. 15, pp. 437-460, 2010.

[14] K. Khrapko and J. Vijg, "Mitochondrial DNA mutations and aging: a case closed?" Nature Genetics, vol. 39, no. 4, pp. 445446, 2007.

[15] K. Ishikawa, K. Takenaga, M. Akimoto et al., "ROSgenerating mitochondrial DNA mutations can regulate tumor cell metastasis," Science, vol. 320, no. 5876, pp. 661664, 2008. 
[16] R. S. Arnold, C. Q. Sun, J. C. Richards et al., "Mitochondrial DNA mutation stimulates prostate cancer growth in bone stromal environment," Prostate, vol. 69, no. 1, pp. 1-11, 2009.

[17] J. A. Petros, A. K. Baumann, E. Ruiz-Pesini et al., "MtDNA mutations increase tumorigenicity in prostate cancer," Proceedings of the National Academy of Sciences of the United States of America, vol. 102, no. 3, pp. 719-724, 2005.

[18] B. Linnartz, R. Anglmayer, and S. Zanssen, "Comprehensive scanning of somatic mitochondrial DNA alterations in acute leukemia developing from myelodysplastic syndromes," Cancer Research, vol. 64, no. 6, pp. 1966-1971, 2004.

[19] A. M. Czarnecka, T. Krawczyk, M. Zdrozny et al., "Mitochondrial NADH-dehydrogenase subunit 3 (ND3) polymorphism (A10398G) and sporadic breast cancer in Poland," Breast Cancer Research and Treatment, vol. 121, no. 2, pp. 511-518, 2010.

[20] A. M. Czarnecka, A. Klemba, T. Krawczyk et al., "Mitochondrial NADH-dehydrogenase polymorphisms as sporadic breast cancer risk factor," Oncology Reports, vol. 23, no. 2, pp. 531-535, 2010.

[21] K. Plak, W. Kukwa, E. Bartnik et al., "The impact of mtDNA mutations on proteins structure in selected types of cancer," Postepy Biochemii, vol. 54, no. 2, pp. 151-160, 2008.

[22] G. Pietka, W. Kukwa, E. Bartnik, A. Ścińska, and A. M. Czarnecka, "Mitochondrial DNA mutations in the pathogenesis in the head and neck squamous cell carcinoma," Otolaryngologia Polska, vol. 62, no. 2, pp. 158-164, 2008.

[23] K. Ishikawa, N. Koshikawa, K. Takenaga, K. Nakada, and J. I. Hayashi, "Reversible regulation of metastasis by ROSgenerating mtDNA mutations," Mitochondrion, vol. 8, no. 4, pp. 339-344, 2008.

[24] S. DiMauro, "Mitochondrial diseases," Biochimica et Biophysica Acta, vol. 1658, no. 1-2, pp. 80-88, 2004.

[25] J. S. Carew and P. Huang, "Mitochondrial defects in cancer," Molecular Cancer, vol. 1, article 9, 2002.

[26] A. M. Czarnecka, A. Klemba, A. Semczuk et al., "Common mitochondrial polymorphisms as risk factor for endometrial cancer," International Archives of Medicine, vol. 2, no. 1, article 33, 2009.

[27] A. M. Czarnecka, T. Krawczyk, K. Plak et al., "Mitochondrial genotype and breast cancer predisposition," Oncology Reports, vol. 24, no. 6, pp. 1521-1534, 2010.

[28] A. Klemba, M. Kowalewska, W. Kukwa et al., "Mitochondrial genotype in vulvar carcinoma-cuckoo in the nest," Journal of Biomedical Science, vol. 17, no. 1, article 73, 2010.

[29] E. Theodoratou, F. V. N. Din, S. M. Farrington et al., "Association between common mtDNA variants and allcause or colorectal cancer mortality," Carcinogenesis, vol. 31, no. 2, pp. 296-301, 2009.

[30] A. A. Kazuno, K. Munakata, T. Nagai et al., "Identification of mitochondrial DNA polymorphisms that alter mitochondrial matrix $\mathrm{pH}$ and intracellular calcium dynamics," PLoS Genetics, vol. 2, no. 8, article e128, pp. 1167-1177, 2006.

[31] B. Baysal, "Mitochondria: more than mitochondrial DNA in cancer," PLoS Medicine, vol. 3, no. 3, article e156, pp. 413414, 2006.

[32] A. S. Don and P. J. Hogg, "Mitochondria as cancer drug targets," Trends in Molecular Medicine, vol. 10, no. 8, pp. 372378, 2004.

[33] C. Vives-Bauza, R. Gonzalo, G. Manfredi, E. Garcia-Arumi, and A. L. Andreu, "Enhanced ROS production and antioxidant defenses in cybrids harbouring mutations in mtDNA," Neuroscience Letters, vol. 391, no. 3, pp. 136-141, 2006.
[34] P. Kakkar and B. K. Singh, "Mitochondria: a hub of redox activities and cellular distress control," Molecular and Cellular Biochemistry, vol. 305, no. 1-2, pp. 235-253, 2007.

[35] Y. Shidara, K. Yamagata, T. Kanamori et al., "Positive contribution of pathogenic mutations in the mitochondrial genome to the promotion of cancer by prevention from apoptosis," Cancer Research, vol. 65, no. 5, pp. 1655-1663, 2005.

[36] G. Amuthan, G. Biswas, S. Y. Zhang, A. Klein-Szanto, C. Vijayasarathy, and N. G. Avadhani, "Mitochondria-tonucleus stress signaling induces phenotypic changes, tumor progression and cell invasion," EMBO Journal, vol. 20, no. 8, pp. 1910-1920, 2001.

[37] M. Kulawiec, H. Arnouk, M. M. Desouki, L. Kazim, I. Still, and K. K. Singh, "Proteomic analysis of mitochondriato-nucleus retrograde response in human cancer," Cancer Biology and Therapy, vol. 5, no. 8, pp. 967-975, 2006.

[38] K. K. Singh, M. Kulawiec, I. Still, M. M. Desouki, J. Geradts, and S. I. Matsui, "Inter-genomic cross talk between mitochondria and the nucleus plays an important role in tumorigenesis," Gene, vol. 354, no. 1-2, pp. 140-146, 2005.

[39] C. Desler, B. Munch-Petersen, T. Stevnsner et al., "Mitochondria as determinant of nucleotide pools and chromosomal stability," Mutation Research, vol. 625, no. 1-2, pp. 112-124, 2007.

[40] K. Ishikawa, O. Hashizume, N. Koshikawa et al., "Enhanced glycolysis induced by mtDNA mutations does not regulate metastasis," FEBS Letters, vol. 582, no. 23-24, pp. 3525-3530, 2008.

[41] A. Salas, Y. G. Yao, V. Macaulay, A. Vega, Á. Carracedo, and H. J. Bandelt, "A critical reassessment of the role of mitochondria in tumorigenesis," PLoS Medicine, vol. 2, no. 11, article e296, pp. 1158-1166, 2005.

[42] H. J. Bandelt, A. Achilli, Q. P. Kong et al., "Low "penetrance" of phylogenetic knowledge in mitochondrial disease studies," Biochemical and Biophysical Research Communications, vol. 333, no. 1, pp. 122-130, 2005.

[43] H. J. Bandelt, Y. G. Yao, A. Salas, T. Kivisild, and C. M. Bravi, "High penetrance of sequencing errors and interpretative shortcomings in mtDNA sequence analysis of LHON patients," Biochemical and Biophysical Research Communications, vol. 352, no. 2, pp. 283-291, 2007.

[44] H. J. Bandelt and A. Salas, "Contamination and sample mixup can best explain some patterns of mtDNA instabilities in buccal cells and oral squamous cell carcinoma," BMC Cancer, vol. 9, article no. 113, 2009.

[45] H. J. Bandelt, P. Lahermo, M. Richards, and V. Macaulay, "Detecting errors in mtDNA data by phylogenetic analysis," International Journal of Legal Medicine, vol. 115, no. 2, pp. 64-69, 2001.

[46] A. Brandstätter, T. Sänger, S. Lutz-Bonengel et al., "Phantom mutation hotspots in human mitochondrial DNA," Electrophoresis, vol. 26, no. 18, pp. 3414-3429, 2005.

[47] A. M. Czarnecka et al., "Methodology for mitochondrial DNA research in oncology: goals and pitfalls," ARS Medica Tomitana, vol. 14, no. 1, pp. 48-64, 2008.

[48] H. J. Bandelt, Y. G. Yao, C. M. Bravi, A. Salas, and T. Kivisild, "Median network analysis of defectively sequenced entire mitochondrial genomes from early and contemporary disease studies," Journal of Human Genetics, vol. 54, no. 3, pp. 174-181, 2009.

[49] Y. G. Yao, C. M. Bravi, and H. J. Bandelt, "A call for mtDNA data quality control in forensic science," Forensic Science International, vol. 141, no. 1, pp. 1-6, 2004. 
[50] V. W. Setiawan, L. H. Chu, E. M. John et al., "Mitochondrial DNA G10398A variant is not associated with breast cancer in African-American women," Cancer Genetics and Cytogenetics, vol. 181, no. 1, pp. 16-19, 2008.

[51] K. Darvishi, S. Sharma, A. K. Bhat, E. Rai, and R. N. K. Bamezai, "Mitochondrial DNA G10398A polymorphism imparts maternal Haplogroup $\mathrm{N}$ a risk for breast and esophageal cancer," Cancer Letters, vol. 249, no. 2, pp. 249255, 2007.

[52] M. P. Mims, T. G. Hayes, S. Zheng et al., "Mitochondrial DNA G10398A polymorphism and invasive breast cancer in African-American women," Cancer Research, vol. 66, no. 3, p. 1880, 2006.

[53] R. M. Andrews, I. Kubacka, P. F. Chinnery, R. N. Lightowlers, D. M. Turnbull, and N. Howell, "Reanalysis and revision of the cambridge reference sequence for human mitochondrial DNA," Nature Genetics, vol. 23, no. 2, p. 147, 1999.

[54] M. Ingman and U. Gyllensten, "mtDB: human mitochondrial genome database, a resource for population genetics and medical sciences," Nucleic Acids Research, vol. 34, pp. D749751, 2006.

[55] E. Ruiz-Pesini, M. T. Lott, V. Procaccio et al., "An enhanced MITOMAP with a global mtDNA mutational phylogeny," Nucleic Acids Research, vol. 35, no. 1, pp. D823-D828, 2007.

[56] J. M. van der Walt, K. K. Nicodemus, E. R. Martin et al., "Mitochondrial polymorphisms significantly reduce the risk of Parkinson disease," American Journal of Human Genetics, vol. 72, no. 4, pp. 804-811, 2003.

[57] J. M. van der Walt, Y. A. Dementieva, E. R. Martin et al., "Analysis of European mitochondrial haplogroups with Alzheimer disease risk," Neuroscience Letters, vol. 365, no. 1, pp. 28-32, 2004.

[58] M. Mancuso, F. L. Conforti, A. Rocchi et al., "Could mitochondrial haplogroups play a role in sporadic amyotrophic lateral sclerosis?" Neuroscience Letters, vol. 371, no. 2-3, pp. 158-162, 2004.

[59] M. Giacchetti, A. Monticelli, I. De Biase et al., "Mitochondrial DNA haplogroups influence the Friedreich's ataxia phenotype," Journal of Medical Genetics, vol. 41, no. 4, pp. 293-295, 2004.

[60] J. A. Canter, A. R. Kallianpur, F. F. Parl, and R. C. Millikan, "Mitochondrial DNA G10398A polymorphism and invasive breast cancer in African-American women," Cancer Research, vol. 65, no. 17, pp. 8028-8033, 2005.

[61] D. Covarrubias, R. K. Bai, L. J. C. Wong, and S. M. Leal, "Mitochondrial DNA variant interactions modify breast cancer risk," Journal of Human Genetics, vol. 53, no. 10, pp. 924-928, 2008.

[62] M. Tanaka, T. Takeyasu, N. Fuku, G. Li-Jun, and M. Kurata, "Mitochondrial genome single nucleotide polymorphisms and their phenotypes in the Japanese," Annals of the New York Academy of Sciences, vol. 1011, pp. 7-20, 2004.

[63] S. R. Krystek, W. J. Metzler, and J. Novotny, "Hydrophobicity profiles for protein sequence analysis," in Current Protocols in Protein Science, chapter 2, unit 2.2, 2001.

[64] R. Grantham, "Amino acid difference formula to help explain protein evolution,” Science, vol. 185, no. 4154, pp. 862-864, 1974.

[65] M. J. Betts and R. B. Russell, "Amino acid properties and consequences of substitutions," in Bioinformatics for Geneticists, I. C. G. Michael and R. Barnes, Eds., pp. 289-316, 2003.
[66] C. Tanford, "The interpretation of hydrogen ion titration curves of proteins," Advances in Protein Chemistry, vol. 17, pp. 69-165, 1962.

[67] M. Gómez-Zaera, J. Abril, L. González et al., "Identification of somatic and germline mitochondrial DNA sequence variants in prostate cancer patients," Mutation Research, vol. 595, no. 1-2, pp. 42-51, 2006.

[68] V. Máximo, P. Soares, J. Lima, J. Cameselle-Teijeiro, and M. Sobrinho-Simões, "Mitochondrial DNA somatic mutations (point mutations and large deletions) and mitochondrial DNA variants in human thyroid pathology: a study with emphasis on Hürthle cell tumors," American Journal of Pathology, vol. 160, no. 5, pp. 1857-1865, 2002.

[69] S. Datta, M. Majumder, N. K. Biswas, N. Sikdar, and B. Roy, "Increased risk of oral cancer in relation to common Indian mitochondrial polymorphisms and autosomal GSTP1 locus," Cancer, vol. 110, no. 9, pp. 1991-1999, 2007.

[70] C. Ye, Y. T. Gao, W. Wen et al., "Association of mitochondrial DNA displacement loop (CA) dinucleotide repeat polymorphism with breast cancer risk and survival among Chinese women," Cancer Epidemiology Biomarkers and Prevention, vol. 17, no. 8, pp. 2117-2122, 2008.

[71] A. Isidoro, E. Casado, A. Redondo et al., "Breast carcinomas fulfill the Warburg hypothesis and provide metabolic markers of cancer prognosis," Carcinogenesis, vol. 26, no. 12, pp. 2095-2104, 2005.

[72] P. Parrella, Y. Xiao, M. Fliss et al., "Detection of mitochondrial DNA mutations in primary breast cancer and fineneedle aspirates," Cancer Research, vol. 61, no. 20, pp. 76237626, 2001.

[73] F. Lopez-Rios, M. Sánchez-Aragó, E. García-Garcí et al., "Loss of the mitochondrial bioenergetic capacity underlies the glucose avidity of carcinomas," Cancer Research, vol. 67, no. 19, pp. 9013-9017, 2007.

[74] L. M. Tseng, P. H. Yin, C. W. Chi et al., "Mitochondrial DNA mutations and mitochondrial DNA depletion in breast cancer," Genes Chromosomes and Cancer, vol. 45, no. 7, pp. 629-638, 2006.

[75] V. Ruppert, D. Nolte, T. Aschenbrenner, S. Pankuweit, R. Funck, and B. Maisch, "Novel point mutations in the mitochondrial DNA detected in patients with dilated cardiomyopathy by screening the whole mitochondrial genome," Biochemical and Biophysical Research Communications, vol. 318, no. 2, pp. 535-543, 2004.

[76] C. W. Liou, T. K. Lin, F. M. Huang et al., "Association of the mitochondrial DNA $16189 \mathrm{~T}$ to $\mathrm{C}$ variant with lacunar cerebral infarction: evidence from a hospital-based casecontrol study," Annals of the New York Academy of Sciences, vol. 1011, pp. 317-324, 2004.

[77] R. Zhang, F. Zhang, C. Wang, S. Wang, Y. -H. Shiao, and Z. Guo, "Identification of sequence polymorphism in the D-Loop region of mitochondrial DNA as a risk factor for hepatocellular carcinoma with distinct etiology," Journal of Experimental and Clinical Cancer Research, vol. 29, no. 1, p. 130, 2010.

[78] A. M. Ray, K. A. Zuhlke, A. M. Levin, J. A. Douglas, K. A. Cooney, and J. A. Petros, "Sequence variation in the mitochondrial gene cytochrome $\mathrm{c}$ oxidase subunit I and prostate cancer in African American men," Prostate, vol. 69, no. 9, pp. 956-960, 2009.

[79] W. Kim, T. K. Yoo, D. J. Shin et al., "Mitochondrial DNA haplogroup analysis reveals no association between the common genetic lineages and prostate cancer in the Korean population," PLoS ONE, vol. 3, no. 5, Article ID e2211, 2008. 
[80] M. S. Lehtonen, J. S. Moilanen, and K. Majamaa, "Increased variation in mtDNA in patients with familial sensorineural hearing impairment," Human Genetics, vol. 113, no. 3, pp. 220-227, 2003.

[81] C. Herrnstadt and N. Howell, "An evolutionary perspective on pathogenic mtDNA mutations: haplogroup associations of clinical disorders," Mitochondrion, vol. 4, no. 5-6, pp. 791798, 2004.

[82] Y. Michikawa, F. Mazzucchelli, N. Bresolin, G. Scarlato, and G. Attardi, "Aging-dependent large accumulation of point mutations in the human mtDNA control region for replication," Science, vol. 286, no. 5440, pp. 774-779, 1999.

[83] K. J. Krishnan, L. C. Greaves, A. K. Reeve, and D. Turnbull, "The ageing mitochondrial genome," Nucleic Acids Research, vol. 35, no. 22, pp. 7399-7405, 2007.

[84] W. H. Pavicic and S. M. Richard, "Correlation analysis between mtDNA 4977-bp deletion and ageing," Mutation Research, vol. 670, no. 1-2, pp. 99-102, 2009.

[85] D. Harman, "Aging: a theory based on free radical and radiation chemistry," Journal of gerontology, vol. 11, no. 3, pp. 298-300, 1956.

[86] A. Trifunovic, A. Wredenberg, M. Falkenberg et al., "Premature ageing in mice expressing defective mitochondrial DNA polymerase," Nature, vol. 429, no. 6990, pp. 417-423, 2004.

[87] C. C. Kujoth, A. Hiona, T. D. Pugh et al., "Medicine: mitochondrial DNA mutations, oxidative stress, and apoptosis in mammalian aging," Science, vol. 309, no. 5733, pp. 481-484, 2005.

[88] A. Trifunovic, A. Hansson, A. Wredenberg et al., "Somatic mtDNA mutations cause aging phenotypes without affecting reactive oxygen species production," Proceedings of the National Academy of Sciences of the United States of America, vol. 102, no. 50, pp. 17993-17998, 2005.

[89] D. Edgar and A. Trifunovic, "The mtDNA mutator mouse: dissecting mitochondrial involvement in aging," Aging, vol. 1, no. 12, pp. 1028-1032, 2009.

[90] A. Hiona, A. Sanz, G. C. Kujoth et al., "Mitochondrial DNA mutations induce mitochondrial dysfunction, apoptosis and sarcopenia in skeletal muscle of mitochondrial DNA mutator mice," PLoS ONE, vol. 5, no. 7, Article ID e11468, 2010.

[91] E. Dufour, M. Terzioglu, F. H. Sterky et al., "Age-associated mosaic respiratory chain deficiency causes trans-neuronal degeneration," Human Molecular Genetics, vol. 17, no. 10, pp. 1418-1426, 2008.

[92] M. D. Brown, E. Starikovskaya, O. Derbeneva et al., "The role of mtDNA background in disease expression: a new primary LHON mutation associated with Western Eurasian haplogroup J," Human Genetics, vol. 110, no. 2, pp. 130-138, 2002.

[93] G. Hudson, V. Carelli, L. Spruijt et al., "Clinical expression of leber hereditary optic neuropathy is affected by the mitochondrial DNA-haplogroup background," American Journal of Human Genetics, vol. 81, no. 2, pp. 228-233, 2007.

[94] Y. Ji, A. M. Zhang, X. Jia et al., "Mitochondrial DNA haplogroups M7b1'2 and M8a affect clinical expression of leber hereditary optic neuropathy in Chinese families with the $\mathrm{m} \cdot 11778 \mathrm{G} \rightarrow \mathrm{A}$ mutation," American Journal of Human Genetics, vol. 83, no. 6, pp. 760-768, 2008.

[95] A. Rosa, B. V. Fonseca, T. Krug et al., "Mitochondrial haplogroup H1 is protective for ischemic stroke in Portuguese patients," BMC Medical Genetics, vol. 9, article 57, 2008.
[96] D. Ghezzi, C. Marelli, A. Achilli et al., "Mitochondrial DNA haplogroup $\mathrm{K}$ is associated with a lower risk of parkinson's disease in Italians," European Journal of Human Genetics, vol. 13, no. 6, pp. 748-752, 2005.

[97] K. Gaweda-Walerych, A. Maruszak, K. Safranow et al., "Mitochondrial DNA haplogroups and subhaplogroups are associated with Parkinson's disease risk in a Polish PD cohort," Journal of Neural Transmission, vol. 115, no. 11, pp. 1521-1526, 2008.

[98] G. Carrieri, M. Bonafè, M. De Luca et al., "Mitochondrial DNA haplogroups and APOE4 allele are non-independent variables in sporadic Alzheimer's disease," Human Genetics, vol. 108, no. 3, pp. 194-198, 2001.

[99] B. Rollins, M. V. Martin, P. A. Sequeira et al., "Mitochondrial variants in schizophrenia, bipolar disorder, and major depressive disorder," PLoS ONE, vol. 4, no. 3, Article ID e4913, 2009.

[100] P. E. Coskun, E. Ruiz-Pesini, and D. C. Wallace, "Control region mtDNA variants: longevity, climatic adaptation, and a forensic conundrum," Proceedings of the National Academy of Sciences of the United States of America, vol. 100, no. 5, pp. 2174-2176, 2003.

[101] R. Saxena, P. I. W. De Bakker, K. Singer et al., "Comprehensive association testing of common mitochondrial DNA variation in metabolic disease," American Journal of Human Genetics, vol. 79, no. 1, pp. 54-61, 2006.

[102] R. K. Bai, S. M. Leal, D. Covarrubias, A. Liu, and L. J. C. Wong, "Mitochondrial genetic background modifies breast cancer risk," Cancer Research, vol. 67, no. 10, pp. 4687-4694, 2007.

[103] L. Shen, J. Wei, T. Chen et al., "Evaluating mitochondrial DNA in patients with breast cancer and benign breast disease," Journal of Cancer Research and Clinical Oncology. In press.

[104] H. Fang, L. Shen, T. Chen et al., "Cancer type-specific modulation of mitochondrial haplogroups in breast, colorectal and thyroid cancer," BMC Cancer, vol. 10, article 421, 2010.

[105] L. M. Booker, G. M. Habermacher, B. C. Jessie et al., "North American white mitochondrial haplogroups in prostate and renal cancer," Journal of Urology, vol. 175, no. 2, pp. 468-472, 2006.

[106] E. E. Mueller, W. Eder, J. A. Mayr et al., "Mitochondrial haplogroups and control region polymorphisms are not associated with prostate cancer in Middle European caucasians," PLoS ONE, vol. 4, no. 7, Article ID e6370, 2009.

[107] X.-Y. Li, Y.-B. Guo, M. Su, L. Cheng, Z.-H. Lu, and D.-P. Tian, "Association of mitochondrial haplogroup D and risk of esophageal cancer in Taihang Mountain and Chaoshan areas in China," Mitochondrion, vol. 11, no. 1, pp. 27-32, 2011.

[108] M. S. Pepe, H. Janes, G. Longton, W. Leisenring, and P. Newcomb, "Limitations of the odds ratio in gauging the performance of a diagnostic, prognostic, or screening marker," American Journal of Epidemiology, vol. 159, no. 9, pp. 882-890, 2004.

[109] L. Xu, Y. Hu, B. Chen et al., "Mitochondrial polymorphisms as risk factors for endometrial cancer in southwest China," International Journal of Gynecological Cancer, vol. 16, no. 4, pp. 1661-1667, 2006.

[110] H. J. Bandelt, A. Salas, and C. M. Bravi, "What is a 'novel'; mtDNA mutation — and does 'novelty' really matter?" Journal of Human Genetics, vol. 51, no. 12, pp. 1073-1082, 2006. 
[111] N. Raule, F. Sevini, A. Santoro, S. Altilia, and C. Franceschi, "Association studies on human mitochondrial DNA: methodological aspects and results in the most common age-related diseases," Mitochondrion, vol. 7, no. 1-2, pp. 29-38, 2007.

[112] J. Yang, Y. Zhu, YI. Tong et al., "The novel G10680A mutation is associated with complete penetrance of the LHON/T14484C family," Mitochondrion, vol. 9, no. 4, pp. 273-278, 2009.

[113] G. De Benedictis, G. Rose, G. Carrieri et al., "Mitochondrial DNA inherited variants are associated with successful aging and longevity in humans," FASEB Journal, vol. 13, no. 12, pp. 1532-1536, 1999.

[114] T. Amo, N. Yadava, R. Oh, D. G. Nicholls, and M. D. Brand, "Experimental assessment of bioenergetic differences caused by the common European mitochondrial DNA haplogroups $\mathrm{H}$ and T," Gene, vol. 411, no. 1-2, pp. 69-76, 2008.

[115] W. A. Beckstead, M. T. W. Ebbert, M. J. Rowe, and D. A. McClellan, "Evolutionary pressure on mitochondrial cytochrome $\mathrm{b}$ is consistent with a role of CytbI7T affecting longevity during caloric restriction," PLoS ONE, vol. 4, no. 6, Article ID e5836, 2009.

[116] S. Suissa, Z. Wang, J. Poole et al., "Ancient mtDNA genetic variants modulate mtDNA transcription and replication," PLoS Genetics, vol. 5, no. 5, Article ID e1000474, 2009.

[117] J. R. Speakman, D. A. Talbot, C. Selman et al., "Uncoupled and surviving: individual mice with high metabolism have greater mitochondrial uncoupling and live longer," Aging Cell, vol. 3, no. 3, pp. 87-95, 2004.

[118] D. C. Samuels, A. D. Carothers, R. Horton, and P. F. Chinnery, "The power to detect disease associations with mitochondrial DNA haplogroups," American Journal of Human Genetics, vol. 78, no. 4, pp. 713-720, 2006.

[119] A. Gómez-Durán, D. Pacheu-Grau, E. López-Gallardo et al., "Unmasking the causes of multifactorial disorders: OXPHOS differences between mitochondrial haplogroups," Human Molecular Genetics, vol. 19, no. 17, pp. 3343-3353, 2010.

[120] D. Martínez-Redondo, A. Marcuello, J. A. Casajús et al., "Human mitochondrial haplogroup $\mathrm{H}$ : the highest VO consumer-is it a paradox?” Mitochondrion, vol. 10, no. 2, pp. 102-107, 2010.

[121] M. J. Jackson, S. Papa, J. Bolaños et al., "Antioxidants, reactive oxygen and nitrogen species, gene induction and mitochondrial function," Molecular Aspects of Medicine, vol. 23, no. 1-3, pp. 209-285, 2002.

[122] R. H. Xu, H. Pelicano, Y. Zhou et al., "Inhibition of glycolysis in cancer cells: a novel strategy to overcome drug resistance associated with mitochondrial respiratory defect and hypoxia," Cancer Research, vol. 65, no. 2, pp. 613-621, 2005.

[123] E. Hervouet, H. Simonnet, and C. Godinot, "Mitochondria and reactive oxygen species in renal cancer," Biochimie, vol. 89, no. 9, pp. 1080-1088, 2007.

[124] I. R. Indran, M. P. Hande, and S. Pervaiz, "Tumor cell redox state and mitochondria at the center of the noncanonical activity of telomerase reverse transcriptase," Molecular Aspects of Medicine, vol. 31, no. 1, pp. 21-28, 2010.

[125] S. J. Ralph, S. Rodríguez-Enríquez, J. Neuzil, E. Saavedra, and R. Moreno-Sánchez, "The causes of cancer revisited: "Mitochondrial malignancy" and ROS-induced oncogenic transformation-why mitochondria are targets for cancer therapy," Molecular Aspects of Medicine, vol. 31, no. 2, pp. 145-170, 2010.
[126] J. Zielonka and B. Kalyanaraman, "“ROS-generating mitochondrial DNA mutations can regulate tumor cell metastasis"- a critical commentary," Free Radical Biology and Medicine, vol. 45, no. 9, pp. 1217-1219, 2008.

[127] W. Ladiges, J. Wanagat, B. Preston, L. Loeb, and P. Rabinovitch, "A mitochondrial view of aging, reactive oxygen species and metastatic cancer," Aging cell, vol. 9, no. 4, pp. 462-465, 2010.

[128] N. G. Larsson, "Somatic mitochondrial DNA mutations in mammalian aging," Annual Review of Biochemistry, vol. 79, pp. 683-706, 2010.

[129] J. Lapointe and S. Hekimi, "Early mitochondrial dysfunction in long-lived Mclk1 mice," The Journal of Biological Chemistry, vol. 283, no. 38, pp. 26217-26227, 2008.

[130] M. V. Blagosklonny, J. Campisi, D. A. Sinclair et al., "Impact papers on aging in 2009," Aging, vol. 2, no. 3, pp. 111-121, 2010.

[131] A. Sanz, D. J. Fernández-Ayala, R. K. Stefanatos, and H. T. Jacobs, "Mitochondrial ROS production correlates with, but does not directly regulate lifespan in Drosophila," Aging, vol. 2, no. 4, pp. 220-223, 2010.

[132] S. E. Schriner, N. J. Linford, G. M. Martin et al., "Medecine: extension of murine life span by overexpression of catalase targeted to mitochondria," Science, vol. 308, no. 5730, pp. 1909-1911, 2005.

[133] D. F. Dai, L. F. Santana, M. Vermulst et al., "Overexpression of catalase targeted to mitochondria attenuates murine cardiac aging," Circulation, vol. 119, no. 21, pp. 2789-2797, 2009.

[134] D. F. Dai, T. Chen, J. Wanagat et al., "Age-dependent cardiomyopathy in mitochondrial mutator mice is attenuated by overexpression of catalase targeted to mitochondria," Aging cell, vol. 9, no. 4, pp. 536-544, 2010.

[135] S. J. Dubec, R. Aurora, and H. P. Zassenhaus, "Mitochondrial DNA mutations may contribute to aging via cell death caused by peptides that induce cytochrome c release," Rejuvenation Research, vol. 11, no. 3, pp. 611-619, 2008.

[136] W. Yang and S. Hekimi, "Two modes of mitochondrial dysfunction lead independently to lifespan extension in Caenorhabditis elegans," Aging Cell, vol. 9, no. 3, pp. 433447, 2010.

[137] D. C. Wallace, "Mitochondrial diseases in man and mouse," Science, vol. 283, no. 5407, pp. 1482-1488, 1999.

[138] D. C. Wallace, "Mitochondria and cancer: warburg addressed," Cold Spring Harbor Symposia on Quantitative Biology, vol. 70, pp. 363-374, 2005.

[139] K. K. Singh and M. Kulawiec, "Mitochondrial DNA polymorphism and risk of cancer," Methods in Molecular Biology, vol. 471, pp. 291-303, 2009.

[140] K. Munakata, M. Tanaka, K. Mori et al., "Mitochondrial DNA $3644 \mathrm{~T} \rightarrow$ C mutation associated with bipolar disorder," Genomics, vol. 84, no. 6, pp. 1041-1050, 2004.

[141] H. R. Elliott, D. C. Samuels, J. A. Eden, C. L. Relton, and P. F. Chinnery, " Pathogenic mitochondrial DNA mutations are common in the general population," American Journal of Human Genetics, vol. 83, no. 2, pp. 254-260, 2008. 


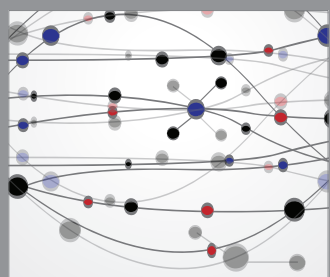

The Scientific World Journal
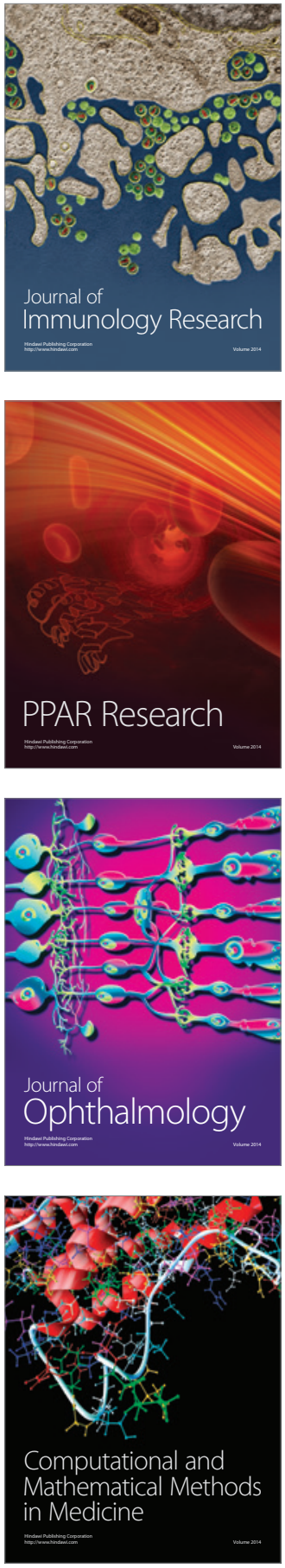

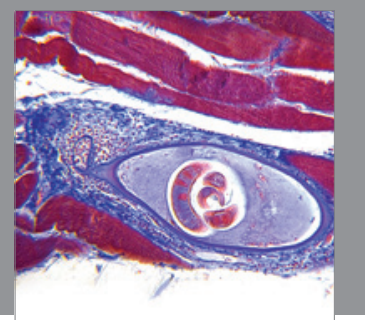

Gastroenterology

Research and Practice
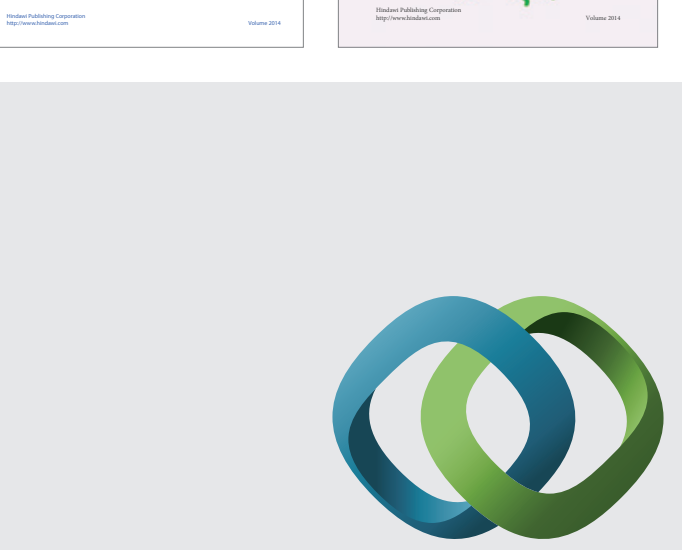

\section{Hindawi}

Submit your manuscripts at

http://www.hindawi.com
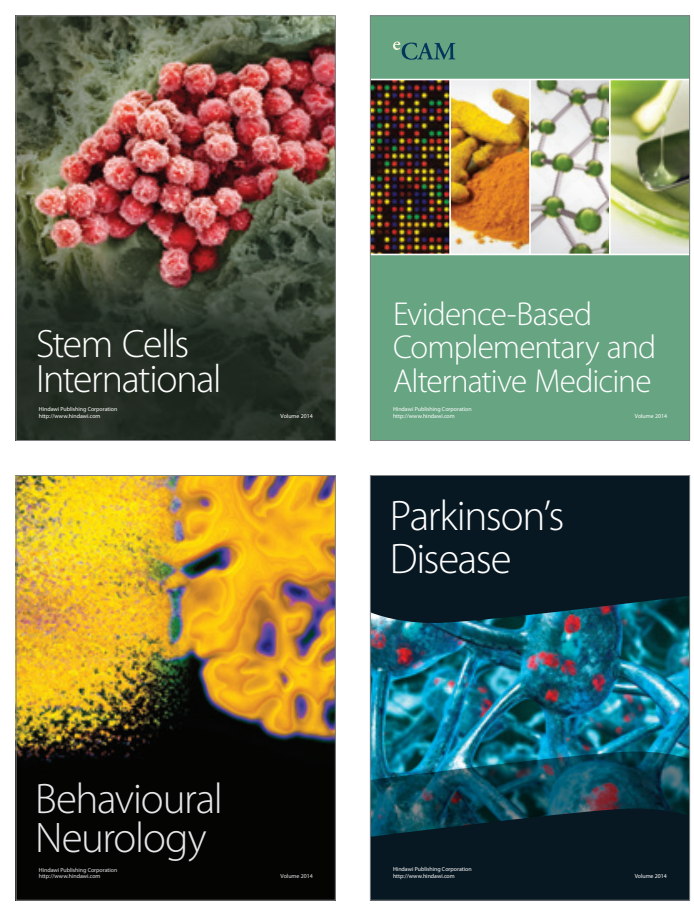

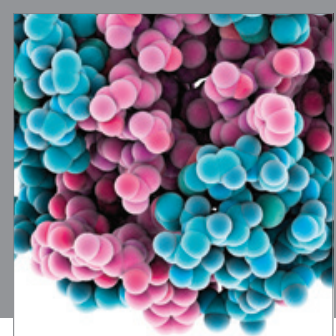

Journal of
Diabetes Research

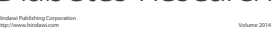

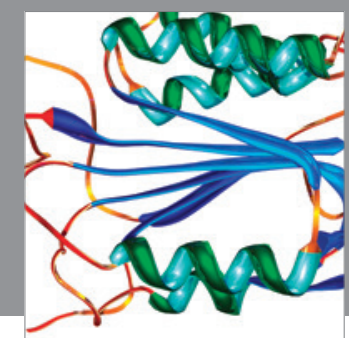

Disease Markers
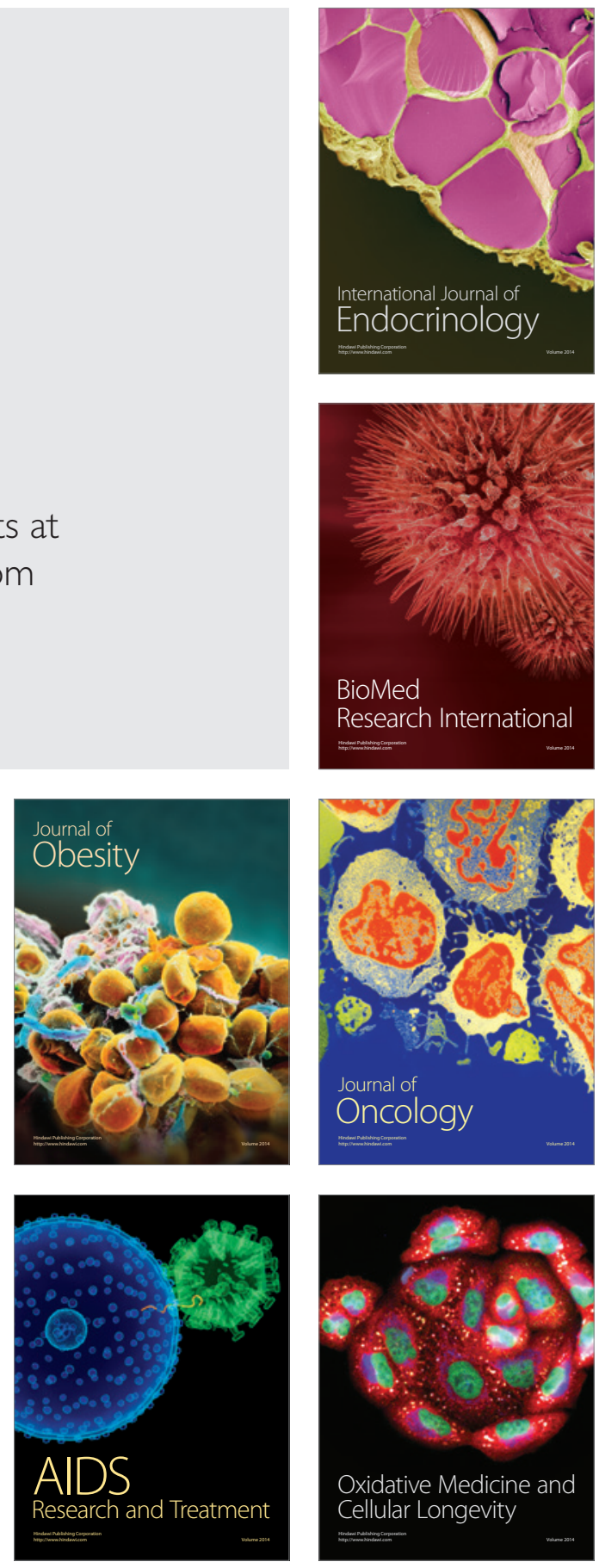